\title{
Pollen Tubes With More Viscous Cell Walls Oscillate at Lower Frequencies
}

\author{
J.H. Kroeger ${ }^{1}$, A. Geitmann ${ }^{2} *$ \\ ${ }^{1}$ Raymor Nanotech, Boisbriand, Québec, Canada \\ ${ }^{2}$ Département de sciences biologiques, Institut de recherche en biologie végétale, Université de \\ Montréal, Montréal, Québec, Canada
}

\begin{abstract}
Pollen tubes are tip growing plant cells that display oscillatory growth behavior. It has been demonstrated experimentally that the reduction of the average pollen tube growth rate through elevated extracellular calcium or borate concentrations coincides with a greater amplitude of the growth rate oscillation and a lower oscillation frequency. We present a simple numerical model of pollen tube growth that reproduces these results, as well as analytical calculations that suggest an underlying mechanism. These data show that the pollen tube oscillator is non-isochronous, and is different from harmonic oscillation.
\end{abstract}

Keywords and phrases: pollen tube growth, oscillations, borate, calcium, harmonic oscillator, modelling

Mathematics Subject Classification: 92C $80,92 \mathrm{C} 10,34 \mathrm{C} 15$

\section{Introduction}

Due to their rich and complex phenomenology as well as the ease with which they lend themselves to in vitro experimentation, pollen tubes have attracted much attention from experimentalists and modellers in the past decade $[21,32]$. Pollen tubes have been referred to as being paradigmatic of tip growing cells due to the apparent simplicity of the mechanism driving growth, i.e. a hydrostatic turgor pressure leading to a strain and subsequent deformation of the cell wall. While their characteristic tubular shape has been reproduced very accurately with different mathematical models, most aspects of pollen tube homeostasis and control have proven subtle and difficult to describe mathematically. One particular phenomenon associated with pollen tube growth has been the object of numerous very different conceptual and mathematical models in the last decade: the oscillation in time of the pollen tube growth rate and other associated variables (ion fluxes and concentrations, vesicle movements and densities, cell wall thickness and rheology, pH) [3]. One reason why so many models have been able to reproduce such oscillations is that (quasi-) sinusoidal oscillations are a common solution of differential equations and models of feedback loops. Simple harmonic oscillation, i.e. the motion of a pendulum or a mass attached to a spring, is perhaps the most studied system in undergraduate physics. It is therefore not surprising that pollen tube growth rate oscillation has been likened to harmonic oscillation [35]. In order to discriminate between many very different models that produce similar oscillation curves, it is important to study the

${ }^{*}$ Corresponding author. E-mail: jkroeger@raymor.com 
changes in oscillation frequency, amplitude and phase resetting under permanent or temporary changes of the growth conditions. One such experimental study investigated the change in oscillation frequency and average growth rate under variable extracellular $\mathrm{pH}$, borate and calcium concentration [13]. These agents are thought to alter the degree of cross-linking between cell wall polymers. A different study applied pectin methyl esterase (PME) to growing pollen tubes, which led to a reduction of the frequency of oscillation [34]. PME removes methyl esters from newly delivered pectin monomers, freeing negatively charged carboxyl groups that can be cross-linked by calcium ions thereby increasing cell wall viscosity. The response of the pollen tube to these changes in the rheological properties of the cell wall material had one commonality, namely that average growth rate and period of oscillation appear to be inversely proportional. Although the reason for this very robust phenomenon is not completely understood, it was speculated that elevated extracellular calcium or borate render the cell wall less extensible, which in turn reduces the average growth rate. If this interpretation is true, and since slower growing pollen tubes display oscillations with lower frequencies, the corollary is that pollen tubes with cell walls of higher viscosity display oscillations with longer periods, i.e. lower frequencies. Here the extensibility is defined as the inverse of the viscosity [5]. Clearly, the pollen tube growth rate oscillates with a frequency that is dependent on the amplitude of the oscillation, i.e. in a non-isochronous manner. By contrast, in simple harmonic motion, amplitude and frequency are independent. In the next section, we present a model whose limit cycle solution is not isochronous in the sense that the period of oscillation depends on its amplitude.

\section{Theory}

Oscillations in the pollen tube growth rate are modelled using a closed feedback loop in a 2-dimensional phase-space. While cell wall strain rate $\dot{\epsilon}$, vesicle exocytosis $R$ and calcium flux $J_{m}$ and $J_{r}$ are incorporated, these quantities can be expressed as a function of apical calcium concentration $C$ and cell wall viscosity $\eta$. The mechanism leading to the oscillations is represented graphically in figure 1 and can be summarized as follows: a slowly varying quantity, the cell wall viscosity, determines the value of the cell wall strain rate, and accordingly the pollen tube growth rate; a quickly varying quantity, the apical calcium concentration, determines the rate of change of the slowly varying quantity, i.e. the cell wall viscosity. A low calcium concentration will, through increased vesicle secretion and the ensuing introduction of soft material into the cell wall, allow the cell wall viscosity to decrease and the cell wall strain rate to increase slowly. At a certain threshold, a sudden calcium influx, triggered by the opening of stretch-activated calcium channels, will quickly increase the calcium concentration and will, by preventing actin polymerization $[16,30]$ and vesicle exocytosis, lead to a cell wall whose viscosity increases by aging. Concomitantly, the cell wall strain rate and the tube growth rate decrease. The negative impact (feedback) of calcium on growth rate is still poorly understood from a biological point of view but has been documented in experimental studies $[16,30]$ and used in feedback models of pollen tube oscillations $[29,33]$. Calcium will eventually be absorbed, leading to the beginning of a new growth cycle.

TABLE 1. Variables used in the model given by equations (2.1)-(2.7).

\begin{tabular}{lc}
\hline \hline Variable & Description \\
\hline$C(\mathrm{mM})$ & apical calcium concentration \\
$\dot{\epsilon}\left(\mathrm{s}^{-1}\right)$ & cell wall strain rate \\
$v\left(\mu m s^{-1}\right)$ & pollen tube growth rate \\
$\eta(\mathrm{MPa} \mathrm{s})$ & cell wall viscosity \\
$R\left(\mu m s^{-1}\right)$ & rate of vesicle exocytosis \\
$J_{m}\left(\mu m \mathrm{~m} \mathrm{~s}^{-1}\right)$ & calcium influx through plasma membrane \\
$J_{r}\left(\mu \mathrm{mm} \mathrm{m} \mathrm{s}^{-1}\right)$ & calcium absorption \\
$D(\dot{\epsilon})\left(\mu \mathrm{m}^{2} \mathrm{~s}^{-1}\right)$ & Diffusion coefficient through calcium channels \\
\hline \hline
\end{tabular}


Specifically, the plastic extension of the cell wall during growth is modelled by an equation relating cell wall strain rate, cell wall stress and cell wall viscosity $(2.1)[4,5,17,26]$. For comparison with experimental data, the growth rate is approximated by multiplying the strain rate $\dot{\epsilon}$ by a characteristic length $L$, as discussed below. The change in calcium concentration in the immediate vicinity of the plasma membrane is proportional to the calcium flux, which is an approximation to the diffusion equation [31]. The apical calcium dynamics (2.2) depend on the influx $J_{m}$ through the stretch-activated calcium channels [6] and a generic absorption term $J_{r}$. In a more refined model, the calcium absorption term $J_{r}$ should depend on the water uptake during growth and therefore be a function of the cell wall strain rate, and possibly the turgor pressure. Osmosis during pollen tube growth is treated in a recent steady-state model of tube growth [12]. The calcium flux through the plasma membrane is given by (2.3), and the diffusion coefficient is a sigmoid function (2.5) of the cell wall strain rate [18,20]. The use of the sigmoid function to describe the gating mechanism of the cell membrane ensures a rapid calcium influx once the strain rate $\dot{\epsilon}$ reaches a critical value [6]. The equation is based on the idea that the calcium channels are stretch-activated and that the stress and strain rates are directly proportional. The cell wall viscosity $\eta$, whose dynamics are described by (2.6), decreases upon delivery of soft cell wall material through vesicle exocytosis $R$, and increases with time due to a generic aging mechanism [7]. This equation for the dynamics of the viscosity is simplistic as it could, in principle, lead to negative values of the viscosity. In practice, the numerical parameters are such that the viscosity always stays positive. These equations were used previously to describe the effect of turgor pressure on pollen tube oscillation frequency and average growth rate [18]. The important change is the inverse dependence of vesicle secretion $R$ on apical calcium concentration $C$. We used the idea, proposed recently [29], that high levels of cytosolic calcium induce the depolymerization of the apical actin cytoskeleton, or actin fringe, and thereby impede the movement, fusion and exocytosis of the new cell wall material, i.e. vesicle secretion. The relation between actin polymerization and calcium concentration was put forth in the model of pollen tube calcium oscillation by Yan et al. [33]. The phenomenological equation (2.7) for the vesicle secretion rate could be refined by considering the effects of vesicle motion as well as both endo- and exocytosis [2].

$$
\begin{aligned}
\dot{\epsilon} & =\frac{1}{\eta}\left(\sigma-\sigma_{y}\right), \\
\frac{\partial C}{\partial t} & =\frac{A}{V_{o l}}\left(J_{m}-J_{r}\right), \\
J_{m} & =D(\dot{\epsilon}) \frac{C_{o}-C}{h}, \\
J_{r} & =a_{C}, \\
D(\dot{\epsilon}) & =\frac{D}{1+e^{-a_{D}\left(\dot{\epsilon}-\dot{\epsilon}_{c}\right)}}, \\
\frac{\partial \eta}{\partial t} & =-\frac{R \eta_{s}}{h}+a_{\eta}\left(\eta_{e q}-\eta\right), \\
R & =\frac{a_{R}}{C} .
\end{aligned}
$$

Here the constants $\sigma$ and $\sigma_{y}$ represent the cell wall stress and the yield stress above which the cell wall behaves plastically. $A$ is the tube tip surface area, $h$ is the cell wall thickness and $V_{o l}$ the tube tip volume. $D$ is the permeability coefficient of the calcium channels and $C_{o}$ is the extracellular calcium concentration. $\eta_{s}$ and $\eta_{e q}$ are the viscosities of the secreted cell wall material and the maximum value attained by the wall due to aging, while $\dot{\epsilon}_{c}$ is the strain rate at which half the stretch-activated channels open. $a_{D}, a_{C}, a_{R}, a_{\eta}$ are rate coefficients for the different reactions. The values of these parameters are given in Table 2. This simple numerical model used to describe the effect of variable cell wall viscosity on the oscillation frequency is based on the works by Kroeger et al. [18,20]. One significant change, based on previous studies $[29,33]$, is the phenomenological expression for the vesicle exocytosis. Instead of being linearly dependent on cytosolic calcium concentration, the vesicle secretion rate is inversely proportional 
to cytosolic calcium concentration. Yan et al. proposed that calcium reduces vesicle exocytosis by depolymerizing the F-actin forming the actin fringe that is responsible for delivering and guiding vesicles towards the site of exocytosis [33]. A schematic representation of the feedback loop responsible for the oscillations in our model is presented in Figure 1. This feedback loop allows to address the effect of a cell wall with a variable viscosity, which was not possible in the original formulation by Kroeger et al. [18,20]. While the original model relied on the cell wall thickness to control the growth rate, in the present model the growth rate is controlled by the cell wall viscosity. By simulating the effect of borate on the dynamics of the cell wall viscosity, the behavior of pollen tubes with variable viscosities can be studied.

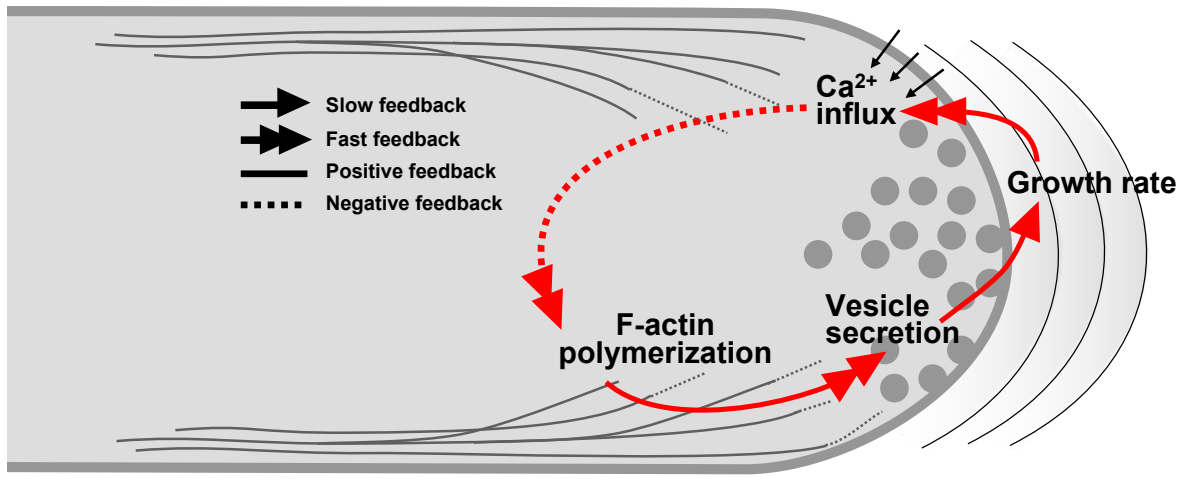

FiguRE 1. Schematic representation of the feedback loop leading to oscillations in the present model. Our model assumes that rapid tube elongation suddenly increases calcium influx through stretch-activated channels (positive and fast feedback). Calcium influx decreases the depolymerization rate of the actin fringe (fast and negative feedback). Actin polymerization activates vesicle secretion (fast and positive feedback) and vesicle secretion gradually lowers the cell wall viscosity and therefore slowly leads to an increase in growth rate (slow and positive feedback). The balance of positive and negative feedback as well as different time scales lead to stable oscillations in the model.

A first order approximation of the oscillation frequency as a function of the average growth rate can be obtained by a slow-fast analysis of the set of coupled equations (2.1)-(2.7). A slow-fast analysis of periodic solutions assumes that during the greatest part of one oscillation cycle, the slow variable varies slowly and the fast variable does not vary at all [28]. The fast variable will vary dramatically during a short fraction of the oscillation cycle, once the slow variable reaches a certain value, thereby activating the fast variable. By separating the growth cycle into a short phase during which the fast variable dominates the dynamics and a long phase during which the slow variable dominates the dynamics, the period of the oscillation is approximated as the time span of the slow phase. For the sake of this analysis, the viscosity $\eta$ is the slow variable and the apical calcium concentration $C$ is the fast variable. The first term on the right hand side of equation (2.6) dominates the dynamics of the viscosity during the slow phase, i.e. when the cytosolic calcium concentration $C$ is close to its basal value and the vesicle secretion rate $R$ is large. In this regime, equation (2.6) can be simplified to

$$
\frac{\partial \eta}{\partial t} \approx \frac{-R \eta_{s}}{h}
$$

and by integrating

$$
\eta(t) \approx \eta(0)-\frac{R \eta_{s}}{h} t,
$$

where $\eta(0)$ is the value of the viscosity at $t=0$. The average strain rate $\langle\dot{\epsilon}\rangle$ is given by

$$
<\dot{\epsilon}>=\Delta \sigma<\eta^{-1}>\text {, }
$$


TABLE 2. Parameterse used in the model given by equations (2.1)-(2.7). The values of the parameters were obtained from experimental studies if available, or otherwise such as to ensure numerical stability $(* *)$.

\begin{tabular}{lcc}
\hline \hline Parameter & Value & Source \\
\hline$\sigma(\mathrm{MPa})$ & 0.3 & {$[4]$} \\
$\sigma_{y}(\mathrm{MPa})$ & 0.1 & {$[4]$} \\
$h(\mu m)$ & 0.2 & {$[25]$} \\
$A\left(\mu m^{2}\right)$ & 36 & {$[11]$} \\
$D\left(\mu m^{2} s^{-1}\right)$ & 300 & $* *$ \\
$C_{o}\left(\mathrm{mM}^{3}\right.$ & 150 & {$[14]$} \\
$V_{o l}\left(\mu m^{3}\right)$ & 216 & {$[11]$} \\
$a_{D}(s)$ & 0.025 & $* *$ \\
$a_{C}\left(\mu m m M s^{-1}\right)$ & 1.4 & {$[18]$} \\
$a_{R}\left(\mu m m M s^{-1}\right)$ & 0.05 & $* *$ \\
$a_{\eta}\left(s^{-1}\right)$ & 0.01 & {$[18]$} \\
$\eta_{e q}(\mathrm{MPa} \mathrm{s})$ & $21-27$ & {$[18]$} \\
$\eta_{s}(\mathrm{MPa} \mathrm{s})$ & 12 & {$[4]$} \\
$\dot{\epsilon}_{c}\left(s^{-1}\right)$ & 0.088 & {$[18]$} \\
\hline \hline
\end{tabular}

where $\Delta \sigma=\sigma-\sigma_{y}$. Furthermore, we use

$$
<\eta^{-1}>=\frac{1}{T} \int_{0}^{T} \frac{d t}{\eta(t)}
$$

where $T$ is the period of one oscillation. By using equation (2.9), we can change our variable of integration and obtain

$$
\frac{1}{T} \int_{0}^{T} \frac{d t}{\eta(t)}=-\frac{h}{R \eta_{s}} \int_{\eta(0)}^{\eta(T)} \frac{d \eta}{\eta}=-\frac{h}{T R \eta_{s}} \cdot[\ln (\eta)]_{n(0)}^{n(T)}
$$

Assuming that at $t=0$, the viscosity has its maximal value $\eta(0)=\eta_{c}+\frac{R T \eta_{s}}{h}$ and that at $t=T$, the viscosity reaches its minimal value which will call $\eta(T)=\eta_{c}$. The constant $\eta_{c}$ can be approximated as $\eta_{c}=\frac{\Delta \sigma}{\dot{\epsilon}_{c}}$, the cell wall viscosity at which half the calcium channels open. We now obtain

$$
<\dot{\epsilon}>\simeq \frac{h \Delta \sigma}{T R \eta_{s}} \ln \left(1+\frac{R \eta_{s} T}{h \eta_{c}}\right) .
$$

As can be seen in Figure 4B, this relation between the strain rate $\langle\dot{\epsilon}\rangle$, previously multiplied by the characteristic length $L$ to obtain a growth rate, and the period of oscillation is a reasonable match to experimental data. The relation bears some resemblance to an inverse proportionality.

\section{Results}

Borate is thought to affect the extensibility of the cell wall by cross-linking rhamnogalacturonan II, a pectic polysaccharide $[9,10,15,24,27]$. Given that pectins are among the major constituents of the pollen tube cell wall [11], and the predominant component in the growing apex [8], a modification of its rheological properties is expected to have significant effects on the growth behavior of the cell. We choose to simulate the effect of borate described by Holdaway et al. (2003) by changing the value of the cell wall viscosity. Changing the cell wall viscosity affects the cell wall extensibility as the extensibility is the inverse of the cell wall viscosity [5]. Since the viscosity is a dynamic quantity, the parameter that is varied is the maximal value that the viscosity will attain due to the aging process. The effect of borate on pollen tube growth was modeled by varying the value of $\eta_{e q}$ in equations (2.1)-(2.7). The value of $\eta_{e q}$ 

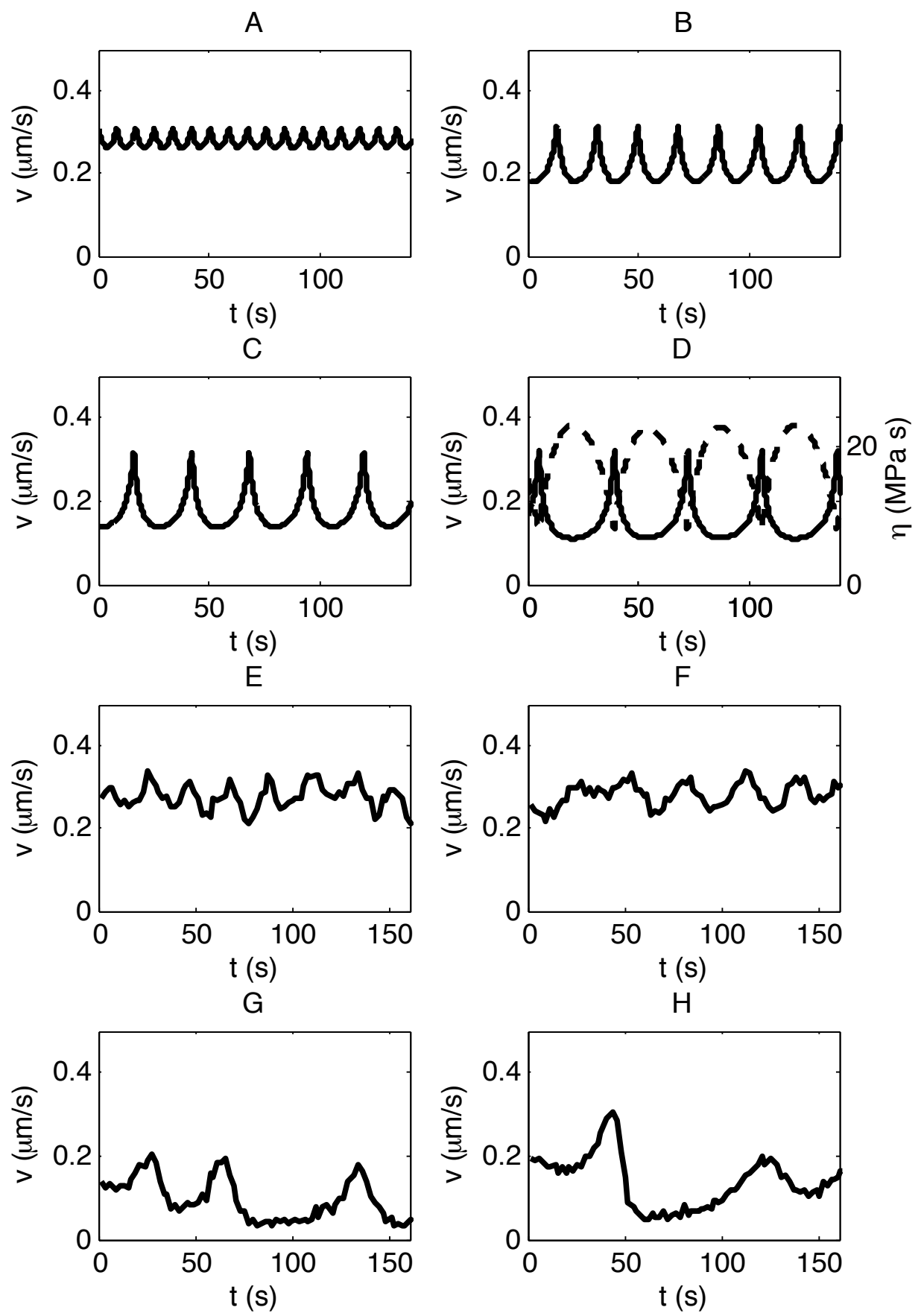

FIGURE 2. Oscillating pollen tube growth rate for different values of the cell wall extensibility. Numerical results A: $\eta_{e q}=24 \mathrm{MPa}$; B: $\eta_{e q}=25,5 \mathrm{MPa}$; C: $\eta_{e q}=27 \mathrm{MPa}$ s; D: $\eta_{e q}=28,5 \mathrm{MPa}$ s. (Dashed line: viscosity $\eta(t)$ in MPa s). Experimental results E,F: Lilium formosanum treated with $3.2 \mathrm{mM}$ of $\mathrm{H}_{3} \mathrm{BO}_{3}$; G,H: Lilium formosanum treated with $16 \mathrm{mM}$ of $\mathrm{H}_{3} \mathrm{BO}_{3}$. Experimental curves are redrawn after Holdaway-Clarke et al. [13]. 
in Eq. (2.6) defines the maximal density of bonds between pectin monomers during the growth cycle and it is this parameter that is varied in Figure 2. This figure compares tube growth rates from numerical simulations (A-D) and experiments (E-H). In both cases, reducing the cell wall extensibility by increasing the numerical parameter $\eta_{e q}$ or applying higher borate concentrations $\left(\mathrm{H}_{3} \mathrm{BO}_{3}\right)$ leads to a decrease in the average growth rate and an increase in both amplitude and period of oscillation. Since the numerical model presented above calculates the strain rate rather than a growth rate, we approximate the growth rate $v$ by multiplying the strain rate $\dot{\epsilon}$ by a characteristic length $L$ such as the length of cell wall which yields to the stress. In the case of tip growth, all of the strain happens over the tip of the cell. We therefore use a value of the order of the tube radius as the characteristic length and obtain $v=L \dot{\epsilon}$ with $L=4 \mu m$.

As seen in Figure 2, the greater the equilibrium viscosity, the lower is the basal pollen tube growth rate. This result can be interpreted as follows: during the "basal growth rate" phase of the growth cycle, the viscosity reaches its equilibrium value and does not change noticeably. According to the constitutive equation (2.1), the strain rate and thus the instantaneous growth rate are inversely proportional to the cell wall viscosity (Figure 2D). As a result, the average growth rate decreases when the equilibrium cell wall viscosity increases (Figure 3A). Concomitantly, the period of oscillation increases (Figure 3B). The explanation for the increase of the period of oscillation upon a decrease in cell wall extensibility (or an increase in equilibrium viscosity $\eta_{e q}$ ) is very similar to the explanation for the change in period upon a change in turgor pressure [18]. Essentially, the period increases because the time needed to attain the critical strain rate is linearly dependent on the difference between basal and maximal growth rates. The further the two are apart, and the longer it takes for the tube to reach the critical growth rate at which massive calcium influx is triggered and the vesicle secretion rate decreases rapidly. It appears that during the slow phase of the growth cycle, the rate of change of the viscosity is approximately constant. This assumption, embodied by equation (2.9), is the main ingredient that leads to the inverse relation between period and average growth rate (Figure 4; equation (2.13)). While the feedback loop proposed here is not the only feedback loop that can produce oscillations $[23,29,33]$, it is the only model that proposes an explanation for the changes in average growth rate and frequency upon changes in turgor [18] or cell wall viscosity.

The main result of the present modelling study, presented in Figure $4 \mathrm{~A}$ and $4 \mathrm{~B}$, is a nearly inverse relation between period and average growth rate. The corollary of this inverse relationship is that the product of the average growth rate and the period of oscillation is nearly constant. For the experimental data set shown in Figure 4C, this product is equal, on average, to 6.5 microns with a standard deviation of 1.9 microns. This product has the dimensions of a distance (i.e. microns) and its value is close to the pollen tube radius. This suggests that, independently of the value of the cell wall viscosity, the growth rate amplitude or frequency, the pollen tube will grow by the same distance during each growth cycle. Whether this is true for other pollen tube species and other treatments is an open question. Rojas et al. suggested that pollen tubes grow by a distance close to their radius during each growth cycle [29], but they did not investigate the dependence of this elongation on cell wall viscosity, period of oscillation, average growth rate, or other parameters. The constant elongation per growth cycle appears to form an ideal subject for future theoretical and experimental studies of pollen tube growth oscillations. The second result of this study is that the growth rate oscillation is non-isochronous, i.e. the frequency is dependent on the amplitude of the oscillation.

\section{Conclusions}

A simple numerical model was presented to explain the effect of cell wall extensibility, through borate application among other means, on the period and average value of the growth rate oscillation. A less extensible (i.e. more viscous) cell wall leads to a lower basal growth rate and, since the maximal growth rate varies very little, to a lower average growth rate. In addition, the amplitude and the period of the oscillation increase when the average growth rate decreases. This behavior demonstrates that the pollen tube oscillator is non-isochronous. Non-isochronous oscillation is different from simple harmonic motion, 

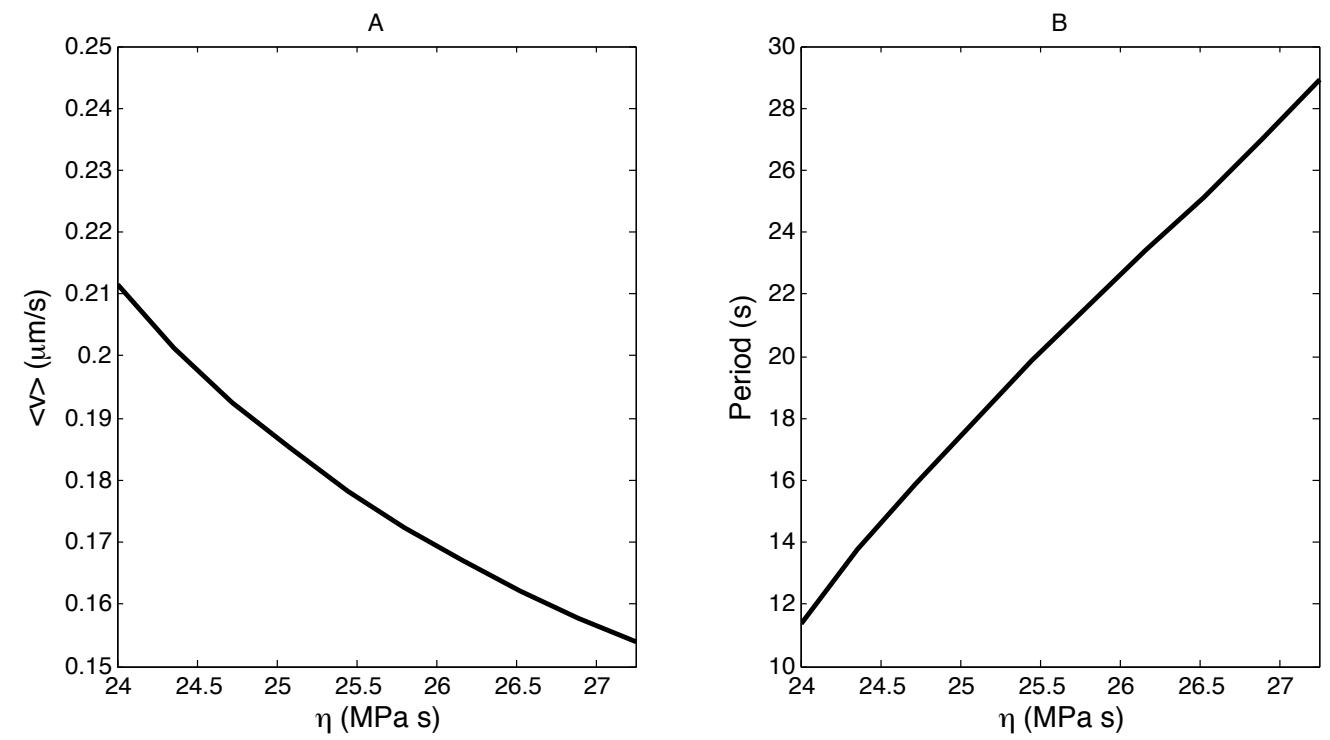

FIgURE 3. A: Average growth rate for different values of the equilibrium viscosity. B: Period of oscillation for different values of the equilibrium viscosity.

i.e. an isochronous oscillation whose frequency is independent of its amplitude. The biological significance of this property for the proper functioning of pollen tube growth is unknown. It can be speculated that, in the presence of many self-sustained and coupled oscillators as may be the case in pollen tube growth, the ability of the different oscillators to adjust their frequency allows them to better synchronize [22], and avoid destructive interference, as would be the case between different non-synchronous oscillators. The synchronicity of different coupled oscillators may, by preventing destructive interference, increase the robustness of the pollen tube oscillation in the repeatedly changing environment to which the elongating pollen tube is exposed while traversing the different pistillar tissues.

Acknowledgements. We would like to thank T. L. Holdaway-Clarke, N. M. Weddle, S. Kim, A. Robi, C. Parris, J. G. Kunkel and P. K. Hepler, as well as the Journal of Experimental Botany, for letting us use their data on the effect of borate on growth oscillations. Research in the Geitmann lab is funded by the Natural Sciences and Engineering Research Council of Canada. 


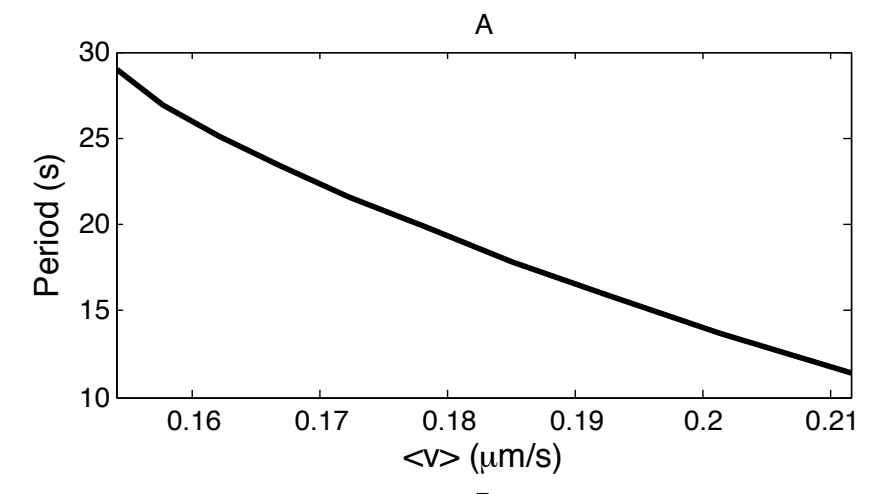

B

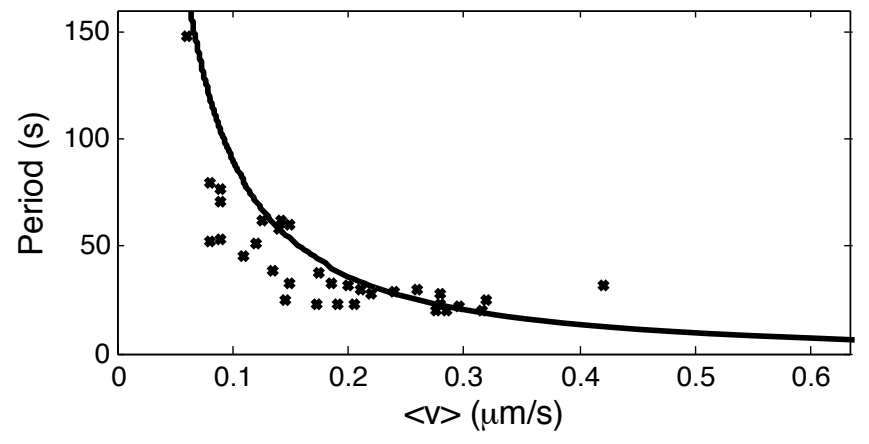

C

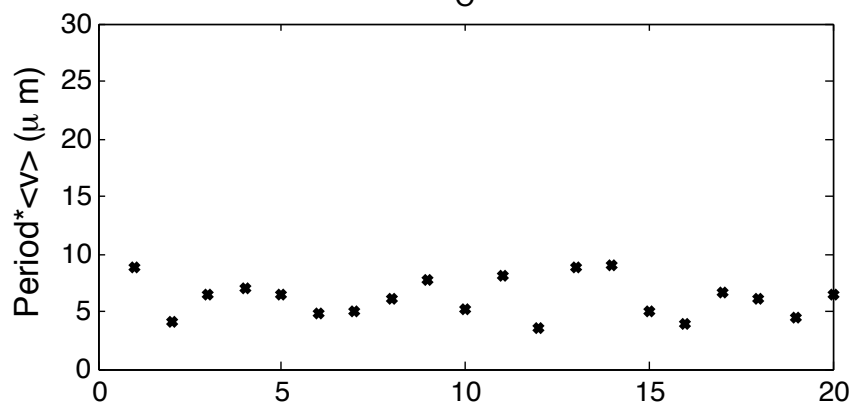

Figure 4. Period of oscillation vs. average pollen tube growth rate. A: Results from the numerical model (equations (2.1)-(2.7)). B: Experimental data from Holdaway-Clarke et al. [13] (black crosses) and theoretical fit to the experimental data using equation (2.13) multiplied by the characteristic length $L$ (black line). C: Product of the period of oscillation and average growth rate (experimental data from Holdaway-Clarke et al. [13]). Here the $\mathrm{y}$-axis represents the number of the data point.

\section{References}

[1] F. Bou Daher, A. Geitmann. Actin is involved in pollen tube tropism through redefining the spatial targeting of secretory vesicles. Traffic, 12 (2011), 1537-1551.

[2] A. Chavarría-Krauser, D. Yejie. A model of plasma membrane flow and cytosis regulation in growing pollen tubes. J. of Theor. Biol., 285 (2011), 10-24.

[3] Y. Chebli, A. Geitmann. Mechanical principles governing pollen tube growth. Functional Plant Science and Biotechnology, 1 (2007), 232-245.

[4] J. Dumais, S.R. Long, S.L. Shaw. The mechanics of surface expansion anisotropy in Medicago truncatula root hairs. Plant Physiology, 136 (2004), 3266-3275. 
[5] J. Dumais, S.L. Shaw, C.R. Steele, S.R. Long, P.M. Ray. An anisotropic-viscoplastic model of plant cell morphogenesis by tip growth. International Journal of Developmental Biology, 50 (2006), 209-222.

[6] R. Dutta, K.R. Robinson. Identification and characterization of stretch-activated ion channels in pollen protoplasts. Plant Physiol., 135 (2004), 1398-1406.

[7] E. Eggen, M.N. de Keijser, B.M. Mulder. Self-regulation in tip-growth: The role of cell wall aging. Journal of Theoretical Biology, 283 (2011), 113-121.

[8] P. Fayant, O. Girlanda, Y. Chebli, C.E. Aubin, I. Villemure, A. Geitmann. Finite element model of polar growth in pollen tubes. Plant Cell, 22 (2010), 2579-2593.

[9] A. Fleischer, C. Titel, R. Ehwald. The boron requirement and cell wall properties of growing and stationary suspensioncultured Chenopidium album L. cells. Plant Physiology, 117 (1998), 1401-1410.

[10] A. Fleischer, M.A. O'Neill, R. Ehwald. The pore size of nongraminaceous plant cell walls is rapidly decreased by borate ester cross-linking of the pectic polysaccharide rhamnogalacturonan II. Plant Physiology, 121 (1999), 829-838.

[11] A. Geitmann, M.W. Steer. The architecture and properties of the pollen tube cell wall. In: R. Malhó (Ed) The Pollen Tube: A Cellular and Molecular. Perspective, Plant Cell Monographs, Springer Verlag, Berlin, 2006.

[12] A.E. Hill, B. Shachar-Hill, J.N. Skepper, J. Powell, Y. Shachar-Hill. An osmotic model of the growing pollen tube. PLoS One, 7 (2012), e36585.

[13] T. L. Holdaway-Clarke, N. M. Weddle, S. Kim, A. Robi, C. Parris, J. G. Kunkel, P. K. Hepler. Effect of extracellular calcium, $p H$ and borate on growth oscillations in Lilium formosanum pollen tubes. Journal of Experimental Botany, 54 (2003), 65-72.

[14] T. L. Holdaway-Clarke, P.K. Hepler. Tansley Review. Control of pollen tube growth: role of ion gradients and fluxes. New Phytologist 159 (2003). 539-563.

[15] T. Ishii, T. Matsunaga, N. Hayashi. Formation of rhamnogalacturonan II-borate dimer in pectin determines cell wall thickness of pumpkin tissue. Plant Physiology, 126 (2001), 1698-1705.

[16] H. Li, Y. Lin , R.M. Heath, M.X. Zhu, Z. Yang. Control of pollen tube growth by a Rop GTPase-dependent pathway that leads to tip-localized calcium influx. Plant Cell, 11 (1999), 1731-1742.

[17] J.A. Lockhart. An analysis of irreversible plant cell elongation. Journal of Theoretical Biology, 8 (1965), $264-275$.

[18] J. H. Kroeger, R. Zerzour, A. Geitmann. Regulator or driving force? The role of turgor pressure in oscillatory plant cell growth. PLoS One, 6 (2011), e18549.

[19] J. H. Kroeger, F. Bou Daher, M. Grant, A. Geitmann. Microfilament orientation constrains vesicle flow and spatial distribution in growing pollen tubes. Biophysical Journal, 97 (2009), 1822-1831.

[20] J.H. Kroeger, A. Geitmann, M. Grant. Model for calcium dependent oscillatory growth in pollen tubes. Journal of Theoretical Biology, 253 (2008), 363-374.

[21] J.H. Kroeger, A. Geitmann. Pollen tube growth: Getting a grip on cell biology through modeling. Mechanical Research Communications, 42 (2012), 32-39.

[22] M. Leoni, T. B. Liverpool. Hydrodynamic synchronisation of non-linear oscillators at low Reynolds number. Phys. Rev. E 85 (2012). 040901.

[23] J. Liu, B.M.A.G. Piette, M.J. Deeks, V. E. Franklin- Tong, P.J. Hussey. A compartmental model analysis of integrative and self-regulatory ion dynamics in pollen tube growth. PLoS One, 5 (2010), e13157.

[24] T. Matoh, M. Kobayashi. Boron and calcium, essential inorganic constituents of pectic polysaccharides in higher plant cell walls. Journal of Plant Research, 111 (1998), 179-190.

[25] S.T. McKenna, J.G. Kunkel, M.B., C.M. Rounds, L. Vidali, L.J. Winship, P.K. Hepler Exocytosis precedes and predicts the increase in growth in oscillating pollen tubes. Plant Cell, 21 (2009), 3026-3040.

[26] J.K.E. Ortega. Governing equations for plant cell growth. Physiologia Plantarum, 79 (1990), $116-121$.

[27] B.L. Ridley, M.A. O'Neill, D.A. Mohnen. Pectins: structure, biosynthesis, and oligogalacturonide-related signaling. Phytochemistry, 57 (2001), 929-967.

[28] J. Rinzel. Bursting oscillations in an excitable membrane model, in ordinary and partial differential equations. In: Sleeman BD, Jarvis RJ, editors. Lecture Notes in Mathematics. (1985) New York: Springer. pp. 304-316.

[29] E. Rojas, S. Hotton, J. Dumais. Chemically mediated mechanical expansion of the pollen tube cell wall. Biophysical Journal, 101 (2011),1844-1853.

[30] S.J. Roy, T.L. Holdaway-Clarke, G.R. Hackett, J.G. Kunkel, E.M. Lord, P.K. Helpler. Uncoupling secretion and tip growth in lily pollen tubes: evidence for the role of calcium in exocytosis. The Plant Journal 19 (1999), 379-386.

[31] G. Ullah, P. Jung, A. Cornell-Bell. Antiphase calcium oscillations in astrocytes via inositol (1,4,5)-trisphosphate regeneration. Cell Calcium, 39 (2006) 197208.

[32] L.J. Winship, G. Obermeyer, A. Geitmann, PK. Hepler. 2010. Under pressure, cell walls set the pace. Trends in Plant Science 15: 363-369.

[33] A. Yan, G. Xu, Z.-B. Yang. Calcium participates in feedback regulation of the oscillating ROP1 Rho GTPase in pollen tubes. P.N.A.S., 106 (2009), 22002-22007.

[34] R. Zerzour, J.H. Kroeger, A. Geitmann. Polar growth in pollen tubes is associated with spatially confined dynamic changes in cell mechanical properties. Dev. Biol., 334 (2009) 437-446.

[35] L. Zonia, T. Munnik. Uncovering hidden treasures in pollen tube growth mechanics. Trends Plant Sci., 14 (2009), 318-327.

[36] L. Zonia, T. Munnik. Vesicle trafficking dynamics and visualization of zones of exocytosis and endocytosis in tobacco pollen tubes. Journal of Experimental Botany, 59 (2008), 861-873. 